Marquette University

e-Publications@Marquette

$10-2008$

\title{
Synthetic studies directed toward amphidinol 2: elucidation of the relative configuration of the $\mathrm{C} 1-\mathrm{C} 10$ fragment
}

Praveen Kommana

Seung Won Chung

William Donaldson

Marquette University, william.donaldson@marquette.edu

Follow this and additional works at: https://epublications.marquette.edu/chem_fac

Part of the Chemistry Commons

\section{Recommended Citation}

Kommana, Praveen; Chung, Seung Won; and Donaldson, William, "Synthetic studies directed toward amphidinol 2: elucidation of the relative configuration of the C1-C10 fragment" (2008). Chemistry Faculty Research and Publications. 47.

https://epublications.marquette.edu/chem_fac/47 
Marquette University

e-Publications@Marquette

\section{Chemistry Faculty Research and Publications/College of Arts and Sciences}

This paper is NOT THE PUBLISHED VERSION; but the author's final, peer-reviewed manuscript. The published version may be accessed by following the link in the citation below.

Tetrahedron Letters, Vol. 49, No. 43 (October 20, 2008): 6209-6211. DOI. This article is (C) Elsevier and permission has been granted for this version to appear in e-Publications@Marquette. Elsevier does not grant permission for this article to be further copied/distributed or hosted elsewhere without the express permission from Elsevier.

\section{Synthetic Studies Directed Toward Amphidinol 2: Elucidation of The Relative Configuration of The C1-C10 Fragment}

Praveen Kommana

Department of Chemistry, Marquette University, Milwaukee WI

Seung Won Chung

Department of Chemistry, Marquette University, Milwaukee WI

William A. Donaldson

Department of Chemistry, Marquette University, Milwaukee WI

\footnotetext{
Abstract

Model compounds (11 and 12) for the C1-C10 tetrahydropyran fragment of amphidinol 2 were prepared from (2S)-benzyloxypropanal in 9 steps. The synthetic route relied on diastereoselective diene-aldehyde cycloaddition, stereoselective C-allylation, and reagent based enantioselective aldehyde allylation. Comparison of the NMR spectra for models $\mathbf{1 1}$ and $\mathbf{1 2}$ with that for amphidinol 2 indicated that the C1-C10 segment of the natural product possesses the $2 R^{*}, 4 R^{*}, 6 R^{*}, 7 S^{*}, 8 R^{*}, 10 S^{*}$ relative configuration.
} 


\section{Graphical abstract}
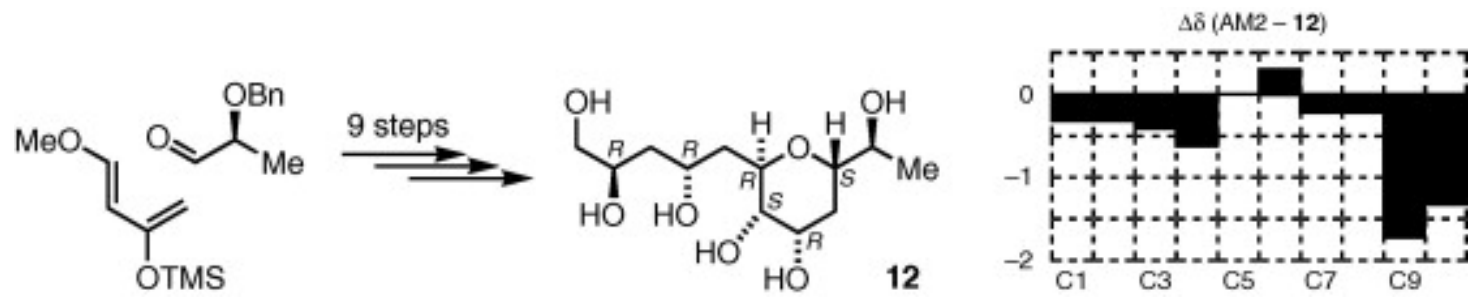

The amphidinols (AM) 1-15 are a series of polyene-polyol natural products isolated from cultured dinoflagellates Amphidinium klebsii and Amphidinium carterae. ${ }^{1}$ The members of this family are characterized by a common bis-pyran polyol segment (highlighted in dashed box for AM2, Fig. 1); they differ with respect to the hydrophobic and hydrophilic chains connected to this common fragment. The amphidinols exhibit variable hemolytic activity as well as antifungal activity against Aspergillus niger (EC50 $=7.3 \mathrm{nM}$ and $6 \mu \mathrm{g} / \mathrm{disk}$, respectively, for $\mathbf{A M} \mathbf{2}^{1 \mathrm{~b}}$ ), and this activity is been attributed to the ability of the amphidinols to increase membrane permeability. It has recently been speculated that the common fragment adopts a 'hairpin' conformation and that the nature of the polyene chain affects the membrane affinity, while differences in the hydrophilic polyol segments of the AMs influence the pore size. ${ }^{2}$ Amphidinol 3 is the only member of this family for which the complete relative and absolute configuration has been determined. ${ }^{3}$ For this reason, amphidinol 3 has attracted the greatest synthetic interest, and numerous groups have prepared extended fragments of this target. $^{4}$

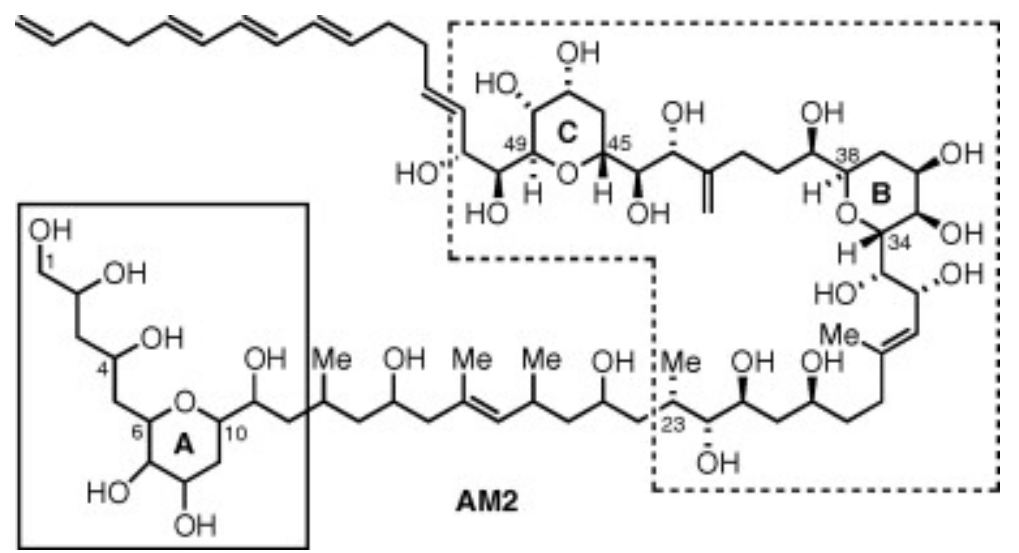

Figure 1. Skeletal structure of amphidinol 2 (AM2).

Amphidinol 2 (AM2) was isolated $>10$ years ago from cultures of Amphidinium klebsii by Tachibana's group. ${ }^{1 \mathrm{~b}}$ The atom connectivity indicated in Figure 1 was assigned on the basis of extensive ${ }^{1} \mathrm{H}$ and ${ }^{13} \mathrm{C}$ NMR spectroscopy. While these authors did not propose the stereochemistry of $\mathbf{A M} 2$ at that time, it now seems likely that the $\mathrm{C} 23-\mathrm{C} 51$ segment of $\mathrm{AM} 2$ has the same relative and absolute configurations as the C23-C51 segment of $A M 3$, given the nearly identical nature of the ${ }^{13} \mathrm{C}$ NMR spectral data for these segments and their similar biological origin. We herein report on synthetic studies directed at elucidating the relative configuration of the C1-C10 segment of AM2 (solid box, Fig. 1).

Tashibana and co-workers ${ }^{1 \mathrm{~b}}$ assigned the hydrogens at $\mathrm{C} 6, \mathrm{C} 7, \mathrm{C} 8$ and $\mathrm{C} 10$ of the tetrahydropyran ring $\mathrm{A}$ as equatorial, equatorial, axial and axial, respectively, on the basis of their ${ }^{3} \mathrm{~J}_{\mathrm{H}-\mathrm{H}}$ couplings. For the purposes of identifying the relative configuration of the $\mathrm{C} 1-\mathrm{C} 10$ segment, we arbitrarily chose to prepare the tetrahydropyran ring with $6 R, 7 S, 8 R, 10 S$ diastereomer. Diastereoselective Lewis acid-catalyzed 
cyclocondensation of 2(S)-benzyloxypropanal (prepared from readily available ethyl (S)-lactate) with 1-methoxy3-(trimethylsiloxy)-1,3-butadiene afforded the known ${ }^{5}$ dihydropyranone $\mathbf{1}$ (Scheme 1). Reduction of 1 gave the pseudoglycal 2. Oxidation of $\mathbf{2}$ with mCPBA in methanol ${ }^{6}$ gave the $\alpha$-methyl 5 -deoxymannoside $\mathbf{3}$, which was protected as its dibenzyl ether $\mathbf{4}$ using $\mathrm{NaH}$ /benzyl bromide. Ionization of the $\alpha$-methoxy group with trimethylsilyl triflate and subsequent nucleophilic attack with allyltrimethylsilane proceeded to give the trans tetrahydropyran 5. $^{7}$ Johnson-Lemieux ${ }^{8}$ oxidation of 5 afforded aldehyde 6.
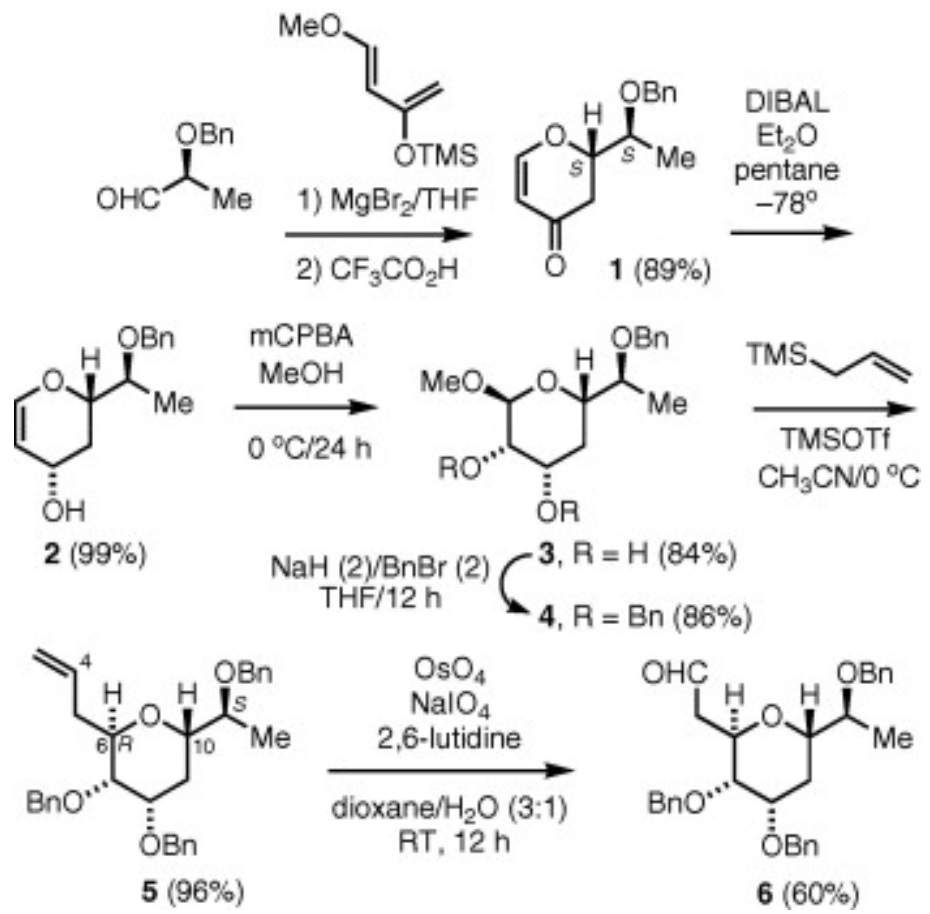

Scheme 1.

Addition of allyl Grignard to $\mathbf{6}$ gave a mixture of two diastereomeric alcohols (7/8), which were difficult to completely separate (Scheme 2). Alternatively, reaction of $\mathbf{6}$ with allyl diisopinocampheylborane ${ }^{9}$ (generated from (+)-(IPC) ${ }_{2} \mathrm{BOMe}$ under salt-free conditions), followed by oxidative work-up, gave $\mathbf{7}$ as the exclusive product. ${ }^{10}$ Homoallylic alcohol 7 was assigned the $4(R)$ stereochemistry on the basis of the ${ }^{1} \mathrm{H}$ NMR spectral data of the corresponding $(S)$ - and $(R)$ - Mosher's esters. In particular, the H-2 signal for the (S)-MTPA ester appears at $\delta 5.62$, while this signal for the (R)-MTPA ester appears upfield at $\delta 5.45 \mathrm{ppm} .{ }^{11}$ In contrast, reaction of 6 with the chiral allylborane generated from (-)-(IPC) 2 BOMe, proceeded in a 'mismatched' double diastereoselective fashion to give a mixture of 7 and $\mathbf{8}$ (1:2.4). Pure $\mathbf{8}^{10}$ could be prepared from pure $\mathbf{7}$ by Mitsunobu inversion ${ }^{12}$ using $p$-nitrobenzoic acid, followed by hydrolysis.
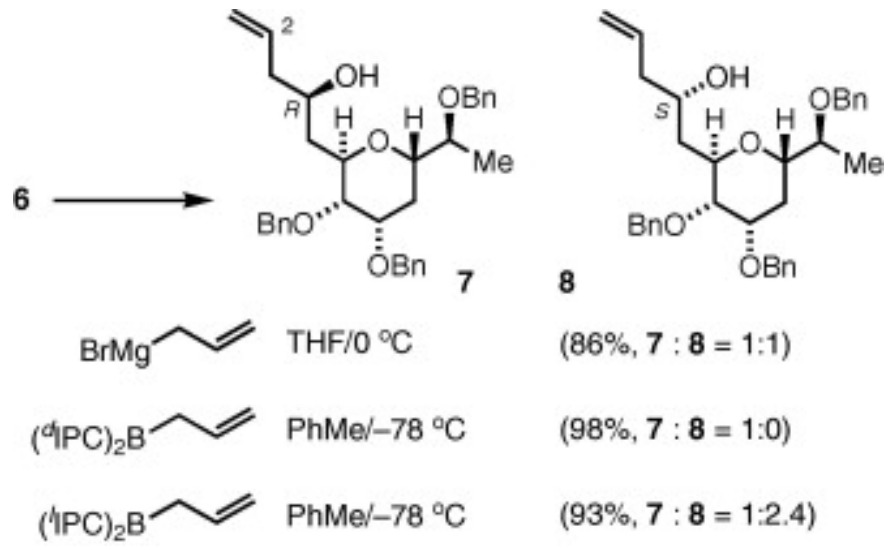


\section{Scheme 2 .}

The ${ }^{13} \mathrm{C}$ NMR spectra of these two diastereomers are relatively similar except for the signals for $\mathrm{C} 4$ and $\mathrm{C} 6$, which appear at $\delta 72.1$ and $76.1 \mathrm{ppm}$ for 7 and $\delta 68.0$ and $71.7 \mathrm{ppm}$ for $\mathbf{8}$, respectively. The downfield shift for these signals in the syn- diastereomer (7) compared to the anti-diastereomer (8) has previously been observed in a number of diastereomeric tetrahydropyran structures bearing an axial (2-hydroxyalkyl)- or (2-hydroxyalkenyl) substituent. ${ }^{13}$ Furthermore, the chemical shift for $\mathrm{C} 6$ of $7(\delta 76.1 \mathrm{ppm})$ is a closer match with that for $\mathrm{C} 6$ of amphidinol 2 ( $\delta 77.3 \mathrm{ppm})$ than is the signal for $\mathrm{C} 6$ of $8(\delta 71.7 \mathrm{ppm})$.

With this insight, dihydroxylation of 7 with $\mathrm{OsO}_{4}$ proceeded in a non-stereoselective fashion to afford a mixture of diols 9 and 10, which were separable by preparative TLC (Scheme 3). The stereochemical assignments for 9 and $\mathbf{1 0}$ (syn- and anti-, respectively) are based on their relative NMR spectral data. ${ }^{10}$ In particular, the sum of the chemical shifts for $\mathrm{C} 2$ and $\mathrm{C} 4$ of $9(\delta 72.0+72.8=144.8 \mathrm{ppm})$ is greater than that for $10(\delta 69.7+70.0=139.7 \mathrm{ppm})$. Hoffmann observed that 'the sum of the chemical shifts of the two oxygen bearing carbon atoms ... should be numerically smaller for the threo-1,3-diols than for their erythrocounterparts' ${ }^{14}$ This difference was attributed to the presence of an axial substituent in the chair-like hydrogen bonded conformers of the erythro-diastereomer, and this empirical trend is documented in numerous cases. ${ }^{15}$

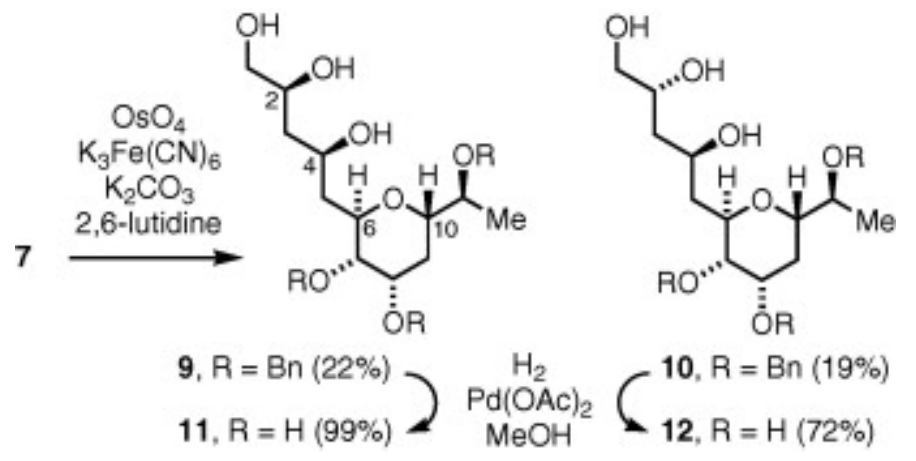

Scheme 3.

Reductive debenzylation of 9 gave 11, while similar processing of 10 gave 12 (Scheme 3). Notably, while the chemical shifts for carbons $\mathrm{C} 6-\mathrm{C} 12$ of $\mathbf{1 1}$ and $\mathbf{1 2}$ are relatively similar, the chemical shifts for $\mathrm{C} 2$ and $\mathrm{C} 4$ of 11 ( $\delta 72.0$ and 69.7$)$ are considerably different than those for 12 ( $\delta 70.4$ and $67.7 \mathrm{ppm})$. A comparison of the ${ }^{13} \mathrm{C}$ NMR signals of $\mathbf{1 1}$ and $\mathbf{1 2}$, obtained in $\mathrm{CD}_{3} \mathrm{OD} / \mathrm{C}_{5} \mathrm{D}_{5} \mathrm{~N} / \mathrm{D}_{2} \mathrm{O}$, with the literature values ${ }^{1 \mathrm{~b}}$ for the corresponding atoms in amphidinol 2 is graphically presented in Figure 2.
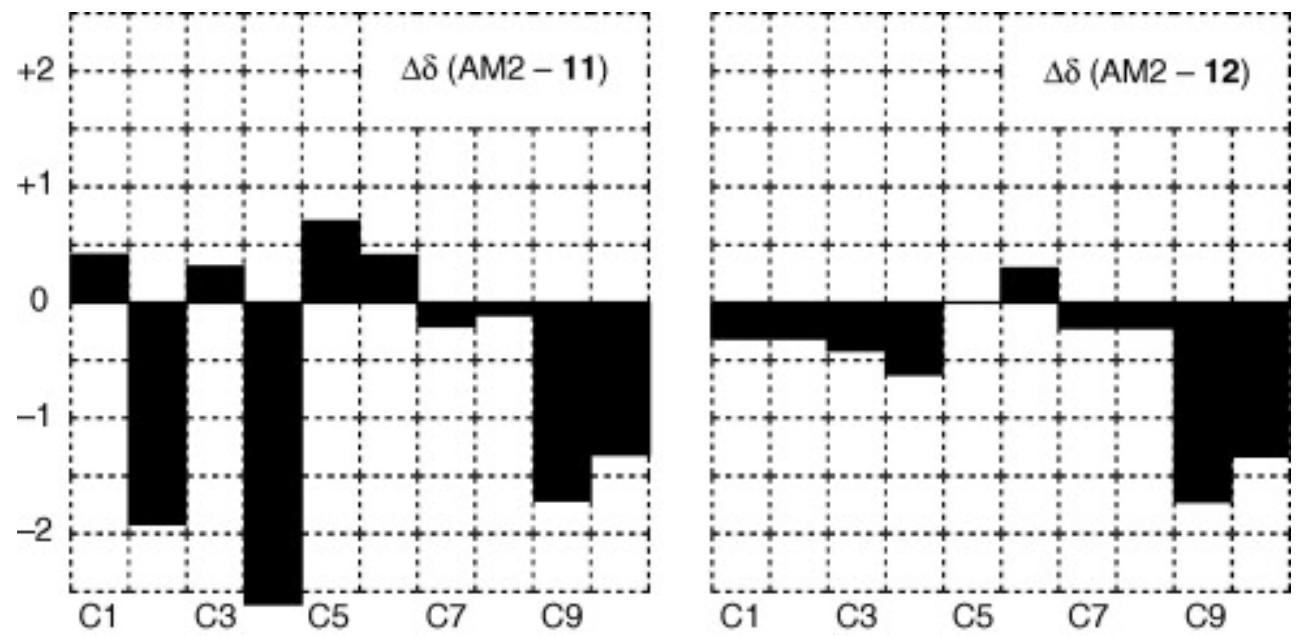

$\begin{array}{lllllll}\mathrm{C} 1 & \mathrm{C} 3 & \mathrm{C} 5 & \mathrm{C} 7 & \mathrm{C} 9\end{array}$ 
Figure 2. Difference between the chemical shifts of the carbon atoms of amphidinol 2 and those of models 11 and $12\left(\mathrm{CD}_{3} \mathrm{OD} / \mathrm{C}_{5} \mathrm{D}_{5} \mathrm{~N} / \mathrm{D}_{2} \mathrm{O}\right)$.

From these comparisons, the chemical shifts of $\mathrm{C} 1-\mathrm{C} 7$ of $\mathbf{1 2}$ have a better match with $\mathbf{A M 2}$, than do those of $11 .{ }^{16}$ Thus, we propose that the relative configuration of $\mathrm{AM} 2$ is $2 R^{*}, 4 R^{*}, 6 R^{*}, 7 S^{*}, 8 R^{*}, 10 S^{*} .{ }^{17}$ The chemical shifts for $\mathrm{C} 9$ and $\mathrm{C} 10$ of both models $\mathbf{1 1}$ and $\mathbf{1 2}$ deviate from those of $\mathbf{A M 2}$ by $>1 \mathrm{ppm}$. From the present studies, it is not clear if these deviations are due to the differences in molecular structure at $\mathrm{C} 12\left[-\mathrm{CH}_{3} \mathrm{Vs}-\right.$ $\mathrm{CH}_{2} \mathrm{CH}(\mathrm{Me}) \mathrm{CH}_{2}$ for $\mathbf{A M 2}$ ] or due to a difference in the relative stereochemistry at $\mathrm{C} 11$ or both. Further studies will be required to establish the relative configuration at $\mathrm{C} 11$ as well as other stereocenters in the polyol chain of AM2 .

Table 1. ${ }^{1} \mathrm{H}$ NMR spectral data for $\mathrm{AM1}, 11$ and 12 [chemical shift in $\delta$, solvent $\mathrm{CD}_{3} \mathrm{OD} / \mathrm{C}_{5} \mathrm{D}_{5} \mathrm{~N} / \mathrm{D}_{2} \mathrm{O}(2: 1: 0.1)$ ]

\begin{tabular}{|l|l|l|l|}
\hline $\mathbf{H}$ & $\mathbf{A M 2}^{\mathbf{a}}$ & $\mathbf{1 1}^{\mathbf{b}}$ & $\mathbf{1 2}^{\mathbf{b}}$ \\
\hline 1 & 3.57 & $3.55-3.63$ & 3.57 \\
\hline $1^{\prime}$ & 3.59 & $3.55-3.63$ & 3.57 \\
\hline 2 & 4.05 & $3.93-4.03$ & 4.06 \\
\hline 3 & 1.66 & $1.64-1.75$ & $1.60-1.71$ \\
\hline $3^{\prime}$ & 1.67 & $1.64-1.75$ & $1.60-1.72$ \\
\hline 4 & 4.14 & 4.08 & 4.15 \\
\hline 5 & 1.68 & $1.64-1.75$ & $1.60-1.71$ \\
\hline $5^{\prime}$ & 2.00 & 1.93 & 1.96 \\
\hline 6 & 4.27 & 4.26 & 4.25 \\
\hline 7 & 3.72 & 3.67 & 3.66 \\
\hline 8 & 4.00 & $3.93-4.03$ & 3.99 \\
\hline
\end{tabular}

a Ref. 1b.

b Present work.

In summary, model compounds 11 and 12 for the C1-C12 segment of amphidinol 2 were prepared in 9 steps from (S)-2-benzyloxypropanal. Comparison of the ${ }^{13} \mathrm{C}$ NMR spectra of these models with that for the corresponding segment of $\mathrm{AM} 2$ indicates that the relative configuration of $\mathrm{AM} 2$ is $2 R^{*}, 4 R^{*}, 6 R^{*}, 7 S^{*}, 8 R^{*}, 10 S^{*}$.

\section{Acknowledgments}

This work was supported by Marquette University through the Wehr Professor Funds and partially supported by NSF (CHE-0415771) and by NSF instrumentation Grant (CHE-0521323). High-resolution mass spectra were obtained at the University of Nebraska-Center for Mass Spectrometry.

\section{References and notes}

1 (a) M. Satake, M. Murata, T. Yasumoto, T. Fujita, H. Naoki, J. Am. Chem. Soc., 113 (1991), pp. 9859-9861 (b) G.K. Paul, N. Matsumori, M. Murata, K. Tachibana, Tetrahedron Lett., 36 (1995), pp. 6279-6282 (c)Paul, G. K.; Matsumori, N.; Konoki, K.; Murata, M.; Tachibana, K. Harmful and Toxic Algal Blooms, Intergovernmental Oceanographic Commission of UNESCO 1996, pp 503-506. (d) G.K. Paul, N. Matsumori, K. Konoki, M. Murata, K. Tachibana, J. Mar. Biotechnol., 5 (1997), pp. 124-128 (g) R. Echigoya, L. Rhodes, Y. Oshima, M. Satake, Harmful Algae, 4 (2005), pp. 383-389 (h) N. Morsy, S. Matsuoka, T. Houdai, N. Matsumori, S. Adachi, M. Murata, T. Iwashita, T. Fujita, Tetrahedron, 61 (2005), pp. 8606-8610 (i) N. Morsy, T. Houdai, S. Matsuoka, N. Matsumori, S. Adachi, T. Oishi, M. Murata, T. Isashita, T. Fujita, Biorg. Med. Chem., 14 (2006), pp. 6548-6554 
2 T. Houdai, S. Matsuoka, N. Morsy, N. Matsumori, M. Satakeb, M. Murata, Tetrahedron, 61 (2005), pp. 27952802

3 M. Murata, S. Matsuoka, N. Matsumori, G.K. Paul, K. Tachibana, J. Am. Chem. Soc., 121 (1999), pp. 870-871

4 (a) S. BouzBouz, J. Cossy, Org. Lett., 3 (2001), pp. 1451-1454 (b) E.M. Flamme, W.R. Roush, Org. Lett., 7 (2005), pp. 1411-1414 (c) J. De Vicente, B. Betzemeier, S.D. Rychnovsky, Org. Lett., 7 (2005), pp. 1853-1856 (d) L.A. Paquette, S.-K. Chang, Org. Lett., 7 (2005), pp. 3111-3114 (e) J.D. Hicks, E.M. Flamme, W.R. Roush, Org. Lett., 7 (2005), pp. 5509-5512 (f) S.-K. Chang, L.A. Paquette, Synlett (2005), pp. 2915-2918 (g) C. Dubost, I.E. Marko, J. Bryans, Tetrahedron Lett., 46 (2005), pp. 4005-4009 (h) J. De Vicente, J. Huckins, S.D. Rychnovsky, Angew. Chem., Int. Ed., 45 (2006), pp. 7258-7262 (i) M.W. Bedore, S.-K. Chang, L.A. Paquette, Org. Lett., 9 (2007), pp. 513-516 (j) J.R. Huckins, J. De Vicente, S.D. Rychnovsky, Org. Lett., 9 (2007), pp. 4757-4760 (k) F. Colobert, T. Kreuzer, J. Cossy, S. Reymond, T. Tsuchiya, L. Ferrie, I.E. Marko, P. Jourdain, Synlett (2007), pp. 2351-2354 (I) J.D. Hicks, W.R. Roush, Org. Lett., 10 (2008), pp. 681-684

5 S.J. Danishefsky, W.H. Pearson, D.F. Harvey, C.J. Maring, J.P. Springer, J. Am. Chem. Soc., 107 (1985), pp. 12561268

6 T. Saleh, G. Rousseau, Tetrahedron, 58 (2002), pp. 2891-2897

7 (a) M.D. Lewis, J.K. Cha, Y. Kishi, J. Am. Chem. Soc., 104 (1982), pp. 4976-4978 (b) I. Paterson, J.G. Cumming, Tetrahedron Lett., 33 (1992), pp. 2847-2850 (c) J.A.C. Romero, S.A. Tabacco, K.A. Woerpel, J. Am. Chem. Soc., 122 (2000), pp. 168-169

8 R. Pappo, D.S. Allen Jr., R.U. Lemieux, W.S. Johnson, J. Org. Chem., 21 (1956), pp. 478-479

9 (a) H.C. Brown, P.K. Jadhav , J. Am. Chem. Soc., 105 (1983), pp. 2092-2093 (b) U.W. Racherla, H.C. Brown, J. Org. Chem., 56 (1991), pp. 401-404

10 Selected spectral data: 7: ${ }^{1} \mathrm{H}$ NMR $\left(300 \mathrm{MHz}, \mathrm{CDCl}_{3}\right) \delta 1.12(\mathrm{~d}, J=6.0 \mathrm{~Hz}, 3 \mathrm{H}), 1.49(\mathrm{td}, J=2.1,15.0 \mathrm{~Hz}, 1 \mathrm{H})$, 1.64-1.81 (m, 2H), $1.99(\mathrm{td}, J=9.3,12.6 \mathrm{~Hz}, 1 \mathrm{H}), 2.10-2.32(\mathrm{~m}, 2 \mathrm{H}), 3.43(\mathrm{t}, J=3.0 \mathrm{~Hz}, 1 \mathrm{H}), 3.68-3.91(\mathrm{~m}$, $4 \mathrm{H}), 4.27(\mathrm{td}, J=3.1,11.7 \mathrm{~Hz}, 1 \mathrm{H}), 4.54(\mathrm{~m}, 2 \mathrm{H}), 4.59(\mathrm{~s}, 2 \mathrm{H}), 4.68(\mathrm{ABq}, J=12.9 \mathrm{~Hz}, 2 \mathrm{H}), 5.04-5.10(\mathrm{~m}$, $2 \mathrm{H}), 5.82$ (tdd, $J=7.2,10.8,16.8 \mathrm{~Hz}, 1 \mathrm{H}), 7.22-7.40(\mathrm{~m}, 15 \mathrm{H}) ;{ }^{13} \mathrm{C} \mathrm{NMR}\left(75 \mathrm{MHz}, \mathrm{CDCl}_{3}\right) \delta 16.0,28.7$, $35.3,41.8,68.0,70.7,71.68,71.73,71.79,73.7,74.1,75.7,76.1,76.6,117.7,127.6,127.7,127.79$, $127.83,128.0,128.1,128.46,128.5,128.6,135.3,138.5,138.7,139.0$. Compound $8:{ }^{1} \mathrm{H}$ NMR (300 MHz, $\left.\mathrm{CDCl}_{3}\right) \delta 1.08(\mathrm{~d}, J=6.0 \mathrm{~Hz}, 3 \mathrm{H}), 1.31(\mathrm{ddd}, J=3.8,8.5,14.6 \mathrm{~Hz}, 1 \mathrm{H}), 1.64-1.80(\mathrm{~m}, 2 \mathrm{H}), 1.94(\mathrm{td}, J=9.9$, $12.8 \mathrm{~Hz}, 1 \mathrm{H}), 2.15(\mathrm{t}, J=6.7 \mathrm{~Hz}, 2 \mathrm{H}), 3.39(\mathrm{t}, J=3.0 \mathrm{~Hz}, 1 \mathrm{H}), 3.55$ (ddd, $J=3.1,6.7,9.5 \mathrm{~Hz}, 1 \mathrm{H}), 3.64-3.77$ $(\mathrm{m}, 3 \mathrm{H}), 4.32(\mathrm{td}, J=3.5,10.7 \mathrm{~Hz}, 1 \mathrm{H}), 4.44(\mathrm{~s}, 2 \mathrm{H}), 4.48(\mathrm{~d}, J=11.5 \mathrm{~Hz}, 1 \mathrm{H}), 4.54(\mathrm{~d}, J=11.5 \mathrm{~Hz}, 1 \mathrm{H}), 4.59$ $(\mathrm{s}, 2 \mathrm{H}), 4.96-5.04(\mathrm{~m}, 2 \mathrm{H}), 5.66$ (tdd, $J=7.2,9.9,17.2 \mathrm{~Hz}, 1 \mathrm{H}), 7.15-7.35(\mathrm{~m}, 15 \mathrm{H}) ;{ }^{13} \mathrm{C} \mathrm{NMR}(75 \mathrm{MHz}$, $\left.\mathrm{CDCl}_{3}\right) \delta 16.0,28.7,35.3,41.7,68.0,70.7,71.68,71.74,71.8,73.7,74.1,75.7,76.5,117.7,127.6,127.7$, $127.8,127.79,127.88,128.0,128.45,128.47,128.48,128.53,135.1,138.2,138.7$,

138.8. Compound 9: ${ }^{1} \mathrm{H}$ NMR $\left(300 \mathrm{MHz}, \mathrm{CDCl}_{3}\right) \delta 1.15(\mathrm{~d}, \mathrm{~J}=6.3 \mathrm{~Hz}, 3 \mathrm{H}), 1.38-1.49(\mathrm{~m}, 2 \mathrm{H}), 1.63$ (td, $J=9.9,14.4 \mathrm{~Hz}, 1 \mathrm{H}), 1.72-1.84(\mathrm{~m}, 2 \mathrm{H}), 1.97(\mathrm{td}, J=8.9,13.0 \mathrm{~Hz}, 1 \mathrm{H}), 3.40(\mathrm{t}, J=3.3 \mathrm{~Hz}, 1 \mathrm{H}), 3.43$ (dd, $J=5.4,11.1 \mathrm{~Hz}, 1 \mathrm{H}), 3.56(\mathrm{dd}, J=3.7,11.1 \mathrm{~Hz}, 1 \mathrm{H}), 3.68(\mathrm{dt}, J=3.5,8.4 \mathrm{~Hz}, 1 \mathrm{H}), 3.76(\mathrm{td}, J=3.6$, $8.7 \mathrm{~Hz}, 1 \mathrm{H}), 3.81-3.91(\mathrm{~m}, 2 \mathrm{H}), 4.06(\mathrm{br} \mathrm{t}, J=9.9 \mathrm{~Hz}, 1 \mathrm{H}), 4.28(\mathrm{brtd}, J=3.0,11.7 \mathrm{~Hz}, 1 \mathrm{H}), 4.49$ $(\mathrm{d}, J=11.1 \mathrm{~Hz}, 1 \mathrm{H}), 4.55(\mathrm{~s}, 2 \mathrm{H}), 4.62(\mathrm{~d}, J=11.1 \mathrm{~Hz}, 1 \mathrm{H}), 4.66(\mathrm{ABq}, J=12.3 \mathrm{~Hz}, 2 \mathrm{H}$ total), 7.23-7.40 (m, $15 \mathrm{H}) ;{ }^{13} \mathrm{C} \mathrm{NMR}\left(75 \mathrm{MHz}, \mathrm{CDCl}_{3}\right) \delta 15.7,28.7,36.7,39.7,66.6,70.9,71.6,72.0,72.1,72.8,73.4,74.2,75.7$, 75.9, 76.8, 127.61, 127.63, 127.79, 127.81, 128.0, 128.1, 128.42, 128.44, 128.46, 128.48, 128.5, 128.54, 138.17, 138.24, 138.3. Compound 10: ${ }^{1} \mathrm{H}$ NMR $\left(300 \mathrm{MHz}, \mathrm{CDCl}_{3}\right) \delta 1.13(\mathrm{~d}, J=6.3 \mathrm{~Hz}, 3 \mathrm{H}), 1.42(\mathrm{br}$ $\mathrm{d}, J=14.7 \mathrm{~Hz}, 1 \mathrm{H}), 1.49-1.66(\mathrm{~m}, 2 \mathrm{H}), 1.74-1.87(\mathrm{~m}, 2 \mathrm{H}), 1.97(\mathrm{td}, J=9.3,12.8 \mathrm{~Hz}, 1 \mathrm{H}), 3.40(\mathrm{t}, J=3.6 \mathrm{~Hz}$, $1 \mathrm{H}), 3.44(\mathrm{dd}, J=6.9,10.8 \mathrm{~Hz}, 1 \mathrm{H}), 3.53(\mathrm{dd}, J=3.6,10.8 \mathrm{~Hz}, 1 \mathrm{H}), 3.69(\mathrm{dt}, J=3.3,8.3 \mathrm{~Hz}, 1 \mathrm{H}), 3.74-3.92$ $(\mathrm{m}, 3 \mathrm{H}), 4.09(\mathrm{dt}, J=3.0,8.3 \mathrm{~Hz}, 1 \mathrm{H}), 4.28(\mathrm{br} \mathrm{d}, J=12.0 \mathrm{~Hz}, 1 \mathrm{H}), 4.52(\mathrm{~d}, J=11.7 \mathrm{~Hz}, 1 \mathrm{H}), 4.55(\mathrm{~s}, 2 \mathrm{H})$, $4.61(\mathrm{~d}, J=11.7 \mathrm{~Hz}, 1 \mathrm{H}), 4.66(\mathrm{ABq}, J=12.3 \mathrm{~Hz}, 2 \mathrm{H}$ total $), 7.22-7.40(\mathrm{~m}, 15 \mathrm{H}) ;{ }^{13} \mathrm{C} \mathrm{NMR}(75 \mathrm{MHz}$, $\left.\mathrm{CDCl}_{3}\right) \delta 15.8,28.7,36.2,39.4,66.8,69.7,70.0,70.9,71.7,72.0,73.5,74.2,75.85,75.9,127.6,127.7$, $127.8,127.9,128.1,128.4,128.5,128.6,138.3,138.4$. Compound $11:{ }^{1} \mathrm{H}$ NMR (400 MHz, $\left.\mathrm{CD}_{3} \mathrm{OD} / \mathrm{C}_{5} \mathrm{D}_{5} \mathrm{~N} / \mathrm{D}_{2} \mathrm{O}=2.0: 1.0: 0.1\right) \delta 1.13(\mathrm{~d}, \mathrm{~J}=6.4 \mathrm{~Hz}, 3 \mathrm{H}), 1.64-1.75(\mathrm{~m}, 3 \mathrm{H}), 1.80-1.97(\mathrm{~m}, 3 \mathrm{H}), 3.51$ (ddd, $J=3.2,6.5,9.6 \mathrm{~Hz}, 1 \mathrm{H}$ ), 3.55-3.63 (m, 2H), 3.67 (br s, $1 \mathrm{H}$ ), 3.83 (pent, $J=6.4 \mathrm{~Hz}, 1 \mathrm{H}$ ), 3.93-4.03 (m, 
$2 \mathrm{H})$, 4.05-4.12 (m, $1 \mathrm{H}), 4.23-4.29(\mathrm{~m}, 1 \mathrm{H}) ;{ }^{13} \mathrm{C} \mathrm{NMR}\left(100 \mathrm{MHz}, \mathrm{CD}_{3} \mathrm{OD} / \mathrm{C}_{5} \mathrm{D}_{5} \mathrm{~N} / \mathrm{D}_{2} \mathrm{O}=\right.$ 2.0:1.0:0.1) $\delta 19.6$, 32.0, 37.9, 41.0, 67.3, 67.4, 69.7, 70.3, 71.8, 72.0, 75.5, 76.9. Compound 12: ${ }^{1} \mathrm{H} \mathrm{NMR}(400 \mathrm{MHz}$, $\left.\mathrm{CD}_{3} \mathrm{OD} / \mathrm{C}_{5} \mathrm{H}_{5} \mathrm{~N} / \mathrm{D}_{2} \mathrm{O}\right) \delta 1.12(\mathrm{~d}, J=6.4 \mathrm{~Hz}, 3 \mathrm{H}), 1.60-1.71(\mathrm{~m}, 4 \mathrm{H}), 1.82(\mathrm{td}, J=10.0,12.8 \mathrm{~Hz}, 1 \mathrm{H}), 1.96$ (ddd, $J=7.4,9.4,14.2 \mathrm{~Hz}, 1 \mathrm{H}$ ), 3.51 (ddd, $J=3.2,6.5,9.7 \mathrm{~Hz}, 1 \mathrm{H}$ ), $3.57(\mathrm{~d}, J=6.0 \mathrm{~Hz}, 2 \mathrm{H}$ ), 3.66

$(\mathrm{t}, J=2.8 \mathrm{~Hz}, 1 \mathrm{H}$ ), 3.83 (pent, $J=6.4 \mathrm{~Hz}, 1 \mathrm{H}), 3.96-4.06(\mathrm{~m}, 2 \mathrm{H}), 4.15$ (pent, $J=6.2 \mathrm{~Hz}, 1 \mathrm{H}), 4.22-4.27(\mathrm{~m}$, $1 \mathrm{H}) ;{ }^{13} \mathrm{C} N M R\left(100 \mathrm{MHz}, \mathrm{CD}_{3} \mathrm{OD} / \mathrm{C}_{5} \mathrm{D}_{5} \mathrm{~N} / \mathrm{D}_{2} \mathrm{O}=2.0: 1.0: 0.1\right) \delta 19.5,32.0,38.5,41.7,67.4,67.7,68.0,70.3$, 70.4, 71.8, 75.5, 77.0.

11 J.A. Dale, H.S. Mosher, J. Am. Chem. Soc., 95 (1973), pp. 512-519

12 O. Mitsunobu, Synthesis (1981), pp. 1-28

13 (a) P.G. Goekjian, T.-C. Wu, Y. Kishi, J. Org. Chem., 56 (1999), pp. 6412-6422, (b)

P. Wolbers, H.M.R. Hoffmann, Tetrahedron, 55 (1999), pp. 1905-1914 (c) P.B. Greer, W.A. Donaldson, Tetrahedron, 58 (2002), pp. 6009-6018 (d) A. Lewis, I. Stefanuti, S.A. Swain, S.A. Smith, R.J.K. Taylor, Org. Biomol. Chem., 1 (2003), pp. 104-116 (e) L.J. Van Orden, B.D. Patterson, S.D. Rychnovsky, J. Org. Chem., 72 (2007), pp. 5784-5793

14 R.W. Hoffmann, U. Weidmann, Chem. Ber., 118 (1985), pp. 3980-3992

15 (a) J.D. Umarye, T. Lessmann, A.B. Garcia, V. Mamane, S. Sommer, H. Waldmann, Chem. Eur. J., 13 (2007), pp. 3305-3319 (b) S. Shang, H. Iwadare, D.E. Macks, L.M. Ambrosini, D.S. Tan, Org. Lett., 9 (2007), pp. 1895-1898 (c) O. Labeeuw, C. Roxhe, P. Pansavath, J.-P. Genet, Org. Lett., 9 (2007), pp. 105-108 (d) J. Murga, J. Garcia-Fortanet, M. Carda, J.A. Marco, J. Org. Chem., 69 (2004), pp. 7277-7283 (e) L. Caggiano, D.J. Fox, D. House, Z.A. Jones, F. Kerr, S. Warren, J. Chem. Soc., Perkin Trans. 1 (2002), pp. 2634-2645 (f) T. Trieselmann, R.W. Hoffmann, K. Menzel, Eur. J. Org. Chem. (2002), pp. 1292-1304 (g) M. Wolberg, W. Hummel, M. Muller, Chem. Eur. J., 7 (2001), pp. 4562-4571 (h) P.T. Bell, B. Dasgupta, W.A. Donaldson, J. Organomet. Chem., 538 (1997), pp. 75-82 (i) H. Kikuzaki, M. Kobayashi, N. Nakatani, Phytochemistry, 31 (1992), pp. 1783-1786 (j) H. Kikuzaki, M. Kobayashi, N. Nakatani, Phytochemistry, 30 (1991), pp. 3647-3651 (k) D.A. Evans, K.T. Chapman, E.M. Carreira, J. Am. Chem. Soc., 110 (1988), pp. 3560-3578

16 While the differences in the ${ }^{1} \mathrm{H}$ NMR spectral data for 11 and 12 (Table 1 ) are not as great as those for ${ }^{13} \mathrm{C}$ NMR spectra, there is also a closer match between $\mathbf{1 2}$ and AM2 than between $\mathbf{1 1}$ and AM2, particularly for the alcohol methine hydrogens $\mathrm{H} 2$ and $\mathrm{H} 4$.

17 The solvent system $\left[\mathrm{CD}_{3} \mathrm{OD} / \mathrm{C}_{5} \mathrm{D}_{5} \mathrm{~N} / \mathrm{D}_{2} \mathrm{O}(2: 1: 0.1)\right]$ used in our work is the same as that used by Tachibana et al. ${ }^{1 b}$ for $\mathbf{A M 2}$. As a referee has noted, this solvent mixture would disrupt intramolecular hydrogen bonding within these polyol structures, and thus should be used with caution should be used when comparing the NMR spectral data in this solvent to those obtained in $\mathrm{CDCl}_{3}$. 